\title{
Association between vitamin C status and CVD and mortality risk in France and Northern Ireland: the PRIME study
}

\author{
N. Lyner ${ }^{1}$, M. C. McKinley ${ }^{1}$, C. C. Patterson ${ }^{1}$, J. W. Yarnell ${ }^{1}$, D. Arveiler ${ }^{2}$, B. Haas ${ }^{2}$, \\ P. Amouyel ${ }^{3}$, J. Dallongeville ${ }^{3}$, J. Ferrières ${ }^{4}$, V. Bongard ${ }^{4}$, F. Kee ${ }^{1}$, A. Bingham ${ }^{5}$, \\ P. Ducimetière ${ }^{5}$ and J. V. Woodside ${ }^{1}$ \\ ${ }^{1}$ Centre of Public Health, Queen's University Belfast, BT12 6BA, ${ }^{2}$ MONICA-Strasbourg, France, ${ }^{3}$ MONICA - Lille, \\ France, ${ }^{4}$ MONICA-Toulouse, France and ${ }^{5}$ INSERM U780, Villejuif, France
}

Vitamin $C$ is an important water-solubleantioxidant ${ }^{(1)}$. Observational prospective cohort studies suggest higher intakes of antioxidants such as vitamin $\mathrm{C}$ are associated with lower cardiovascular disease (CVD) and mortality risk ${ }^{(2)}$, although fewer studies have examined plasma vitamin $\mathrm{C}$ status. The aim of this study was to examine the relation between plasma vitamin C and CVD incidence and all cause mortality in the PRIME prospective cohort study.

The PRIME study was designed to examine the substantial differences in cardiovascular incidence and mortality between Northern Ireland and France. The PRIME study recruited men aged 50-59 y during 1991-1993 from Belfast, Northern Ireland $(n=2748)$, and Lille $(n=2627)$, Strasbourg $(n=2611)$, and Toulouse $(n=2610)$ in France. Blood samples were taken from participants at baseline, stabilised and frozen at $-150^{\circ} \mathrm{C}$. Participants were followed-up annually. Over 10 years of follow-up, $<5 \%$ of men were lost to followup, while 6.2\% $(n=654)$ men died, and 5.2\% $(n=546)$ had a CVD event, and these events were validated by a medical committee.

Vitamin C was measured on a BMG FLUOstar Optima plate reader. Cox's Proportional Hazard Regression was used to determine the association between plasma vitamin $\mathrm{C}$ and risk of overall mortality and CVD incidence; $p$-values were determined using a trend test across the four quartiles of plasma vitamin C. Men who had CHD or CVD at entry to the study were excluded from analysis

\begin{tabular}{|c|c|c|c|c|c|c|c|}
\hline \multirow{2}{*}{$\frac{\text { Endpoint }}{\text { Overall mortality }}$} & \multirow{2}{*}{$\frac{\text { Quar-tile }}{1}$} & \multicolumn{2}{|l|}{ Unadjusted HR(CI) } & \multicolumn{2}{|c|}{$\begin{array}{l}\text { HR adjusted for country and } \\
\text { age }(\mathrm{CI})\end{array}$} & \multicolumn{2}{|c|}{ HR adjusted for all variables $(\mathrm{CI}) \dagger$} \\
\hline & & 1.00 & & 1.00 & & 1.00 & \\
\hline & 2 & $0.55(0.42-0.73)$ & & $0.58(0.44-0.76)$ & & $0.66(0.50-0.88)$ & \\
\hline & 3 & $0.48(0.36-0.64)$ & $p<0.001^{*}$ & $0.49(0.37-0.65)$ & $p<0.001^{*}$ & $0.59(0.44-0.80)$ & $p=0.01 *$ \\
\hline & 4 & $0.49(0.37-0.65)$ & & $0.51(0.38-0.68)$ & & $0.63(0.47-0.85)$ & \\
\hline \multirow[t]{4}{*}{ CVD incidence } & 1 & 1.00 & & 1.00 & & 1.00 & \\
\hline & 2 & $0.77(0.53-1.12)$ & $p=0.03^{*}$ & $0.81(0.56-1.17)$ & $p=0.04^{*}$ & $0.85(0.59-1.24)$ & $p=0.26$ \\
\hline & 3 & $0.60(0.40-0.88)$ & & $0.61(0.41-0.91)$ & & $0.69(0.46-1.02)$ & \\
\hline & 4 & $0.70(0.48-1.02)$ & & $0.72(0.50-1.05)$ & & $0.86(0.58-1.26)$ & \\
\hline
\end{tabular}

HR - hazard ratio, CI - 95\% confidence interval, *denotes significant p values $<0.05$.

$\dagger$ Adjusted for country, age, systolic blood pressure, diabetes, BMI, cholesterol, HDL cholesterol, height, total physical activity, alcohol and smoking.

After full adjustment for potential confounders, there was a significant inverse association between vitamin $\mathrm{C}$ status and risk of overall mortality. There was no significant association between plasma vitamin C and CVD incidence in the fully adjusted model, however HR trends were in the expected directions and significant associations were observed in the unadjusted model, and also when adjusted for country and age.

In conclusion, higher plasma vitamin $\mathrm{C}$ concentrations were associated with reduced risk of overall mortality, but not CVD incidence, after adjustment for potential confounders. If this association is causal, increasing vitamin $\mathrm{C}$ status in the population could contribute to reduced rates of mortality.

1. Riccioni G (2012) Int J VitamNutr Res 82, 15-26.

2. Kizhakekuttu TJ \& Widlansky ME (2010) CardiovascTher 28, 20. 